\title{
Comparing viewer and array mental rotations in different planes
}

\author{
MACKENZIE CARPENTER and DENNIS R. PROFFITT \\ University of Virginia, Charlottesville, Virginia
}

\begin{abstract}
Participants imagined rotating either themselves or an array of objects that surrounded them. Their task was to report on the egocentric position of an item in the array following the imagined rotation. The dependent measures were response latency and number of errors committed. Past research has shown that self-rotation is easier than array rotation. However, we found that imagined egocentric rotations were as difficult to imagine as rotations of the environment when people performed imagined rotations in the midsagittal or coronal plane. The advantages of imagined self-rotations are specific to mental rotations performed in the transverse plane.
\end{abstract}

When confronted with an object or scene that must be construed from some perspective other than their immediate viewpoint, people are able to imagine rotations of two sorts. They can choose to mentally rotate themselves or to rotate the object or array. Here is an example of the first sort: "Imagine that you are in your kitchen, facing the sink. Turn $270^{\circ}$ to your left. What is in front of you?" Alternatively, the problem could be posed in a manner that requires that the array be rotated: "Again, face the sink, and now imagine that the kitchen spins around you to the right, $270^{\circ}$. What is in front of you this time?"

Considerable research bolsters the prediction that you found the first task to be easier than the second. It has been repeatedly shown that people can more successfully imagine themselves rotating within an environment than imagine the same environment rotating around them (Amorim \& Stucchi, 1997; Huttenlocher \& Presson, 1979; Presson, 1982; Wraga, Creem, \& Proffitt, 1999, 2000). This difference is notable, in part, because the visual transformations that underlie the two rotations are identical.

Whereas recent research has focused on comparing imagined environmental and observer rotations in the horizontal (transverse) plane, none has compared performance in these conditions for imagined rotations in other planes. It may be that the advantage found for mentally rotating oneself as opposed to the world is specific to imagining rotations that are physically possible to execute. There may also be something special about the perpendicular relationship of the array to the principal axis of the observer that allows for easier imagined egocentric rotations around this axis. The present studies compared

We thank Sarah Creem and Melissa Dixon for assistance in data analysis and generation of ideas. This research was supported by NIMH Grant MH52640, NASA Grant NCC2925, and CMU/DARPA Grant 539689-52273 to D.R.P. Correspondence should be addressed to D. R. Proffitt, Psychology Department, University of Virginia, P. O. Box 400400, Charlottesville, VA 22904-4400 (e-mail: drp@ virginia. edu). mental rotation performance in the transverse, coronal, and midsagittal planes and found that the advantage of viewer rotations is limited to the transverse plane. When people performed similar imagined rotations in the coronal or midsagittal plane, the viewer advantage disappeared. Before presenting these studies, we briefly describe the issues and prior research that motivated their design.

\section{Spatial Reference Frames}

When perceiving their own location and the location of objects in the environment, people make use of different spatial reference frames: the object-relative frame, the egocentric frame, and the environmental frame. An object or array has an object-relative frame composed of the up/down, right/left, and front/back orientations internal to the object or array. In the present study, we are concerned with arrays of objects. The egocentric reference frame codes the up/down, right/left, and front/back positions of an observer. Finally, the environmental reference frame is defined by the gravitational up/down and by either permanent landmarks or by compass directions relative to some point.

Imagined viewer and array rotations entail differences with respect to the spatial reference frame that is transformed. In the case of a viewer rotation, the object-relative and environmental frames remain stationary as the person imagines his/her body's frame rotating. For an array rotation, the egocentric and environmental frames remain in place while the intrinsic frame of the array is rotated around its center.

\section{Imagined Viewer Rotations Are Easier Than Imagined Array Rotations in the Horizontal Plane}

Several findings indicate that people perform imagined viewer rotations more quickly and accurately than imagined single-object or multiple-object rotations (Amorim \& Stucchi, 1997; Huttenlocher \& Presson, 1979; Presson, 1982; Wraga et al., 2000). Huttenlocher and Pres- 
son (1979) compared 7-year-old children's performance on imagined array and viewer rotations. They used the following stimuli: a cube painted different colors on each side, a room painted with the same four colors, one on each wall, and a diamond-shaped array of four distinct objects presented on a table. Participants imagined themselves (in the viewer task) or the array (in the array task) rotating $0^{\circ}, 90^{\circ}, 180^{\circ}$, or $270^{\circ}$, and then indicated which side or object had a given relation to them (i.e., front, back, right, or left). Participants responded to the viewer rotation questions more quickly and accurately than the array questions when positioned inside the array (in the room array task) and outside of the array (with the cube, and the four-object array). Presson (1982) replicated these results with adult participants.

In a recent study, Wraga et al. (2000) extended the investigation of the viewer advantage finding to a variety of new situations. They used Presson's (1982) diamondshaped array paradigm, but constructed a life-sized configuration of four objects placed on pedestals. Wraga et al. (2000) replicated Presson's results, with participants standing in front of or in the center of the array. However, array/object performance improved when the object's parts were more cohesive. Wraga et al. (2000) suggested that the viewer advantage was attributable to the fact that the body's frame could be mentally rotated more holistically than an array of objects. They hypothesized that this ability may have evolved from people's experience locomoting in a primarily static environment. In addition, they suggested that the difference in task performance could indicate the use of distinct neural mechanisms for each task. Other researchers have also postulated that a dissociation may exist between the neural mechanism used during manipulations of the egocentric reference frame and the mechanism used to mentally alter the environmental frame (Amorim \& Stucchi, 1997; Milner \& Goodale, 1995; Shepard, 1994). Current neural imaging research has focused on describing the neural processes controlling mental rotations (Alivisatos \& Petrides, 1996; Cohen et al., 1996; Kosslyn, DiGirolamo, Thompson, \& Alpert, 1998; Parsons et al., 1995).

\section{Is the Imagined Viewer Rotation Advantage a General Finding?}

In every experiment comparing viewer and array task performance, observers executed imagined rotations only in the horizontal (transverse) plane. In addition, the array itself was always positioned in the participants' transverse plane. There are good reasons to suspect that the advantage for viewer rotations in the transverse plane is a special case. Natural locomotion is physically possible only on inclines that deviate little from the transverse plane, and consequently, people's experience is constrained to these movements. It may be that the advantage of viewer rotations is due to what is physically possible, or consequently, to what has frequently occurred in experience. Instead, it may be that maintaining a transverse relationship between the viewer and the imagined plane of rotation is all that is needed to demonstrate a viewer advantage.

In the present study, we compare the relative difficulty of viewer and array rotations around the participant's three major axes, these being orthogonal to the participant's coronal, transverse, and median sagittal planes (Figure 1). We hypothesized that the viewer bias evident in performance of transverse-plane rotations would diminish if the required imagined rotations were not performed in the transverse plane with respect to the observer.

The experimental design was a variation of Huttenlocher and Presson's (1979) task. In one of three experiments, participants stood in the center of a life-sized diamond-shaped array of four objects located in their transverse (Experiment 1), coronal (Experiment 2), or midsagittal (Experiment 3) plane. They were instructed to imagine themselves rotating within the array or to imagine the array rotating around them a given number of degrees in the specified plane. Participants were then
X-Z or Transverse Plane

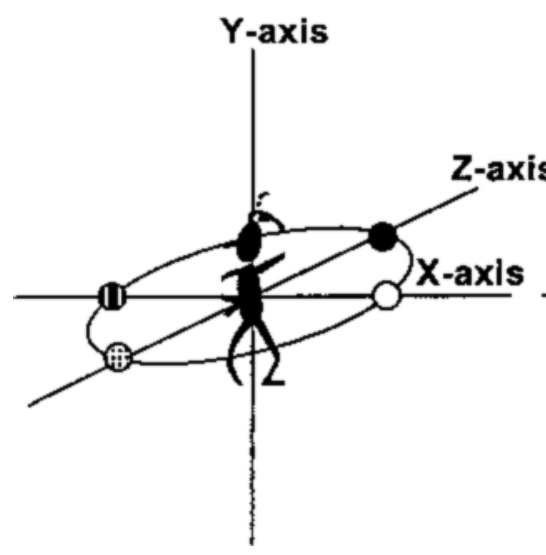

\section{X-Y or Coronal Plane}

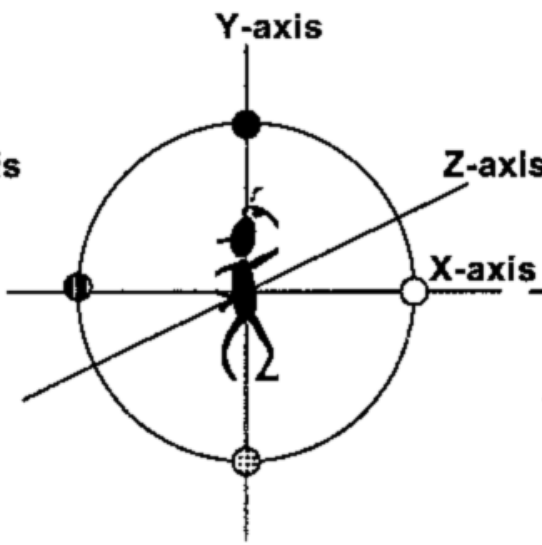

\section{Y-Z or Sagittal Plane}

Figure 1. Experimental planes of mental rotations.

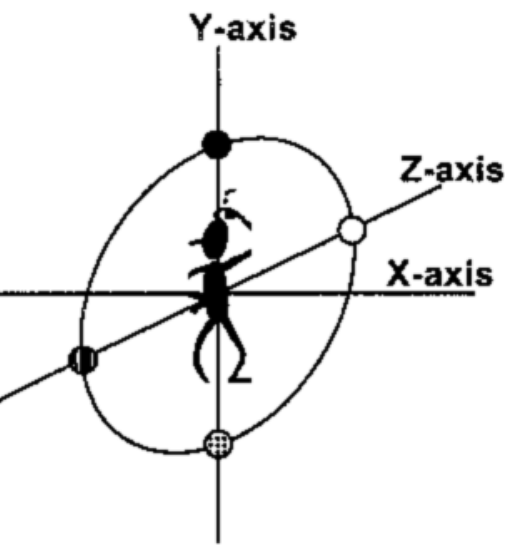


asked to name one of the objects on the basis of its location relative to their imagined position. The dependent measures were response accuracy and latency.

Replicating the results of previous studies, a viewer advantage was found in Experiment 1 (transverse array) for both dependent measures. However, there was no viewer advantage in either Experiment 2 (coronal array) or Experiment 3 (sagittal array). These findings indicate that the advantage for manipulating the egocentric reference frame over the object-relative frame is evident only when the observer performs a transverse-plane imagined rotation.

\section{GENERAL METHOD}

Three experiments were conducted, each comparing viewer and array performance in the transverse, coronal, or median sagittal plane, respectively. This section describes the methods common to all three experiments.

\section{Materials}

The experimental context consisted of an array of four spherical foam balls, $7.5 \mathrm{~cm}$ in diameter, of different colors: red, yellow, green, and blue. The balls were suspended from a $229-\mathrm{cm}$ ceiling with Stitch-Thru invisible thread to form a diamond-shaped array, $151 \mathrm{~cm}$ on a side, with a diagonal extent of $214 \mathrm{~cm}$. The orientation of the array with respect to the participant varied by experiment. Reaction times (RTs) were recorded using a Timex chronograph stopwatch.

\section{Participants}

Twenty-four University of Virginia undergraduates participated in each experiment. All participants received research credit in an undergraduate psychology course. They were unaware of the experimental hypothesis and were tested individually.

\section{Procedure}

Participants stood in the center of the array with their feet stationary and were given an opportunity to become familiar with the balls by looking around and behind them. The balls were identified for the participants by their color and position relative to the participant.

Participants were given the time necessary to learn the positions of each colored ball in the array. They were then quizzed with their eyes closed to ensure that they had learned the array successfully. If not, participants were given the opportunity to learn the array again until they could recall the position of the four colored balls accurately. Each participant performed in both the viewer and array tasks, and the balls' positions were reordered for each experiment.

In the viewer task, participants were asked to imagine themselves rotating in the center of the array a given number of degrees $\left(0^{\circ}\right.$, $90^{\circ}, 180^{\circ}$, or $270^{\circ}$ ) and were then asked to name a ball at a particular position (right, left, top, bottom, front, or back, depending on the experiment) relative to their imagined orientation. The format of each trial question was "number of degrees, what is on/in the position?" In the array task, participants were asked to imagine their bodies remaining stationary and the array of balls rotating around its center. Again, participants were given a position and asked to name the color of the ball in that position once the mental rotation of the array was complete. Participants were asked to imagine themselves (in the viewer task) or the array (in the array task) back in the starting position between trials.

RTs were recorded from the end of the question asked by the experimenter to the beginning of the participant's answer. The first response given by the participant was always recorded, and no feedback was provided to participants regarding performance. After the first array or viewer task was complete, the blindfold was removed and the participants were given a 5-min break while the positions of the balls in the array were rearranged. Participants then completed the remaining task.

\section{Design}

In each experiment, a within-subjects design was employed, and the order of tasks (array, viewer) and rotation directions (right/left, clockwise/counterclockwise, or forward/backward) were counterbalanced across participants. They performed both tasks in a single direction of rotation. In each task, participants completed a total of 16 trials, which exhausted all possible combinations of color (red, yellow, green, or blue) and angle $\left(0^{\circ}, 90^{\circ}, 180^{\circ}\right.$, or $\left.270^{\circ}\right)$.

\section{Analyses}

RTs and number of errors were recorded. Applying a log transformation to the latency data created a symmetrical distribution. A square root transformation was used on the error data to remove positive skew. ${ }^{1}$ Two mixed-design analyses of variance (ANOVAs) were performed on the log transformation latency data and the square root transformation error data: a 2 (direction of rotation) $\times 2$ (task order $) \times 2($ sex $) \times 2$ (task) $\times 4$ (degree) ANOVA and a 2 (rotation direction) $\times 2$ (task order) $\times 2$ (sex) $\times 2$ (task) $\times 2$ (position) mixeddesign ANOVA using SPSS-Macintosh procedures. The latter ANOVA was performed in order to determine whether there was any effect of position (front, back, top, bottom, right, and left) on the latency or accuracy results.

\section{EXPERIMENT 1}

Experiment 1 replicated previous viewer and array mental rotation studies in the horizontal plane (Huttenlocher \& Presson, 1979; Presson, 1982; Wraga et al., 2000). Participants performed imagined egocentric and environmental frame rotations in the center of a four-object array located in their transverse plane.

\section{Method}

Participants. Twenty-four undergraduates participated (13 females, 11 males). Three additional participants were excluded from the study because they responded incorrectly to more than $50 \%$ of the test questions in one or more tasks, and 2 participants were excluded for not following instructions.

Materials. The spheres forming the stimulus array all hung $107 \mathrm{~cm}$ from the floor to form a diamond-shaped array (Figure 1).

Procedure. Participants stood with their heels on a marker positioned directly in the center of the array, facing one of the colored balls, so that the array surrounded them in their transverse plane. The positions of the balls relative to the participants were front, back, right, and left. In the viewer task, participants imagined themselves rotating to their right or to their left in the center of the array. In the array task, participants imagined the array of objects rotating around them in a circle, either to their left or to their right.

\section{Results and Discussion}

In tasks requiring imagined rotations in the horizontal plane, participants responded faster and made fewer errors when they imagined themselves rotating in the environment as opposed to imagining objects in the environment rotating around them. In addition, a position comparison revealed an advantage for front/back responses over right/left responses.

Latency. Participants performed viewer tasks significantly faster than array tasks in the transverse plane. RTs increased with an increase in angle of imagined ro- 
tation during both tasks. A log transformation graph of the mean RTs and standard errors for correct responses is plotted as a function of degrees of rotation in panel a of Figure 2. For each degree of rotation, mean RT outliers ( $3 S D$ from the group mean RT within each task) were replaced with the group mean. One array RT was replaced. Overall latency was greater in the array task $(M=2.89 \mathrm{sec})$ than in the viewer task $(M=1.77 \mathrm{sec})$. The ANOVA performed on the log transformation of the mean RTs revealed a main effect of task $[F(1,16)=26.02$, $p<.0001]$ and degree $[F(3,48)=57.73, p<.0001]$, as well as a significant task $\times$ degree interaction $[F(3,48)=$ $7.97, p<.0001]$. More specifically, the viewer and array RTs were not different at $0^{\circ}$, but the overall latency of array responses was greater than that of viewer responses for $90^{\circ}, 180^{\circ}$ and $270^{\circ}$ of rotation.

The pattern of RT results in the array task replicated previous horizontal plane findings (Cooper, 1975; Shepard \& Metzler, 1971), indicating a positive direct relationship between the degrees of imagined rotation, and the RTs of the participant's accurate responses. Some of these studies did not require participants to imagine object rotations beyond $180^{\circ}$ (Shepard \& Cooper, 1982; Shepard \& Metzler, 1971). However, more recent research employing the same paradigm as that used here produced the same pattern of results: RTs increased linearly from $0^{\circ}$ to $270^{\circ}$ (Presson, 1982; Wraga et al., 2000).

Errors. Fewer mistakes were made in the viewer task $(M=0.45$ errors $)$ than in the array task $(M=0.89$ errors). Mean errors are plotted as a function of degrees of rotation in panel c of Figure 3. The ANOVA performed on the number of errors indicated a main effect of task $[F(1,16)=7.14, p=.017]$ and degree $[F(3,48)=17.00$, $p<.0001]$. There was a significant task $\times$ degree interaction $[F(3,48)=6.78, p=.001]$, and comparison analysis indicated a difference in errors only at $270^{\circ}$ of rotation, where number of errors decreased as compared with $180^{\circ}$ in the viewer task, but where number of errors increased sharply as compared with $180^{\circ}$ in the array task.

Position comparisons. RTs and number of errors were collapsed over positions relative to the location of the participant after a given imagined rotation (right/left and top/bottom) for the array and viewer tasks. This was done in order to observe whether any latency or error rates were greater for particular positions, since previous research indicated a natural advantage for identifying objects in top, bottom, front, and back positions as opposed to right and left positions (Franklin \& Tversky, 1990).

Latency. Right/left position responses were significantly slower than front/back responses in both the viewer task [right $/$ left, $M=2.01 \mathrm{sec}$; front $/ \mathrm{back}, M=1.5 \mathrm{sec}$; $F(1,23)=15.61, p=.001]$ and the array task [right/left, $M=3.46 \mathrm{sec} ;$ front $/ \mathrm{back}, M=2.52 \mathrm{sec} ; F(1,23)=15.14$, $p=.001]$.

Errors. There were a greater number of errors for the right/left positions ( $M=2.04$ errors) in the array task than for the front/back positions $[M=1.50$ errors; $F(1,23)=5.72, p<.025]$. No position effect was found for the viewer task (right/left, $M=1.04$ errors; front/ back, $M=0.71$ errors).

\section{EXPERIMENT 2}

In Experiment 2, participants performed array and viewer mental rotations on objects forming an array in their coronal or picture plane. The experiment was designed to determine whether the viewer advantage revealed in Experiment 1 was also evident when participants performed mental rotations in a plane in which people cannot naturally locomote, in this case, the coronal plane.

\section{Method}

Participants. Twenty-four undergraduates participated (12 females, 12 males). Two additional participants were excluded from the study because they responded incorrectly to more than $50 \%$ of the test questions in one or more tasks, and 1 participant was excluded for not following instructions.

Materials. Two of the spheres forming the stimulus array were suspended $107 \mathrm{~cm}$ from the floor, one ball rested on the floor, and the fourth hung $214 \mathrm{~cm}$ directly over it.

Procedure. Participants stood with their feet on either side of the sphere placed on the floor, with a ball to each side, and one overhead so that the objects were all located in their coronal plane. The

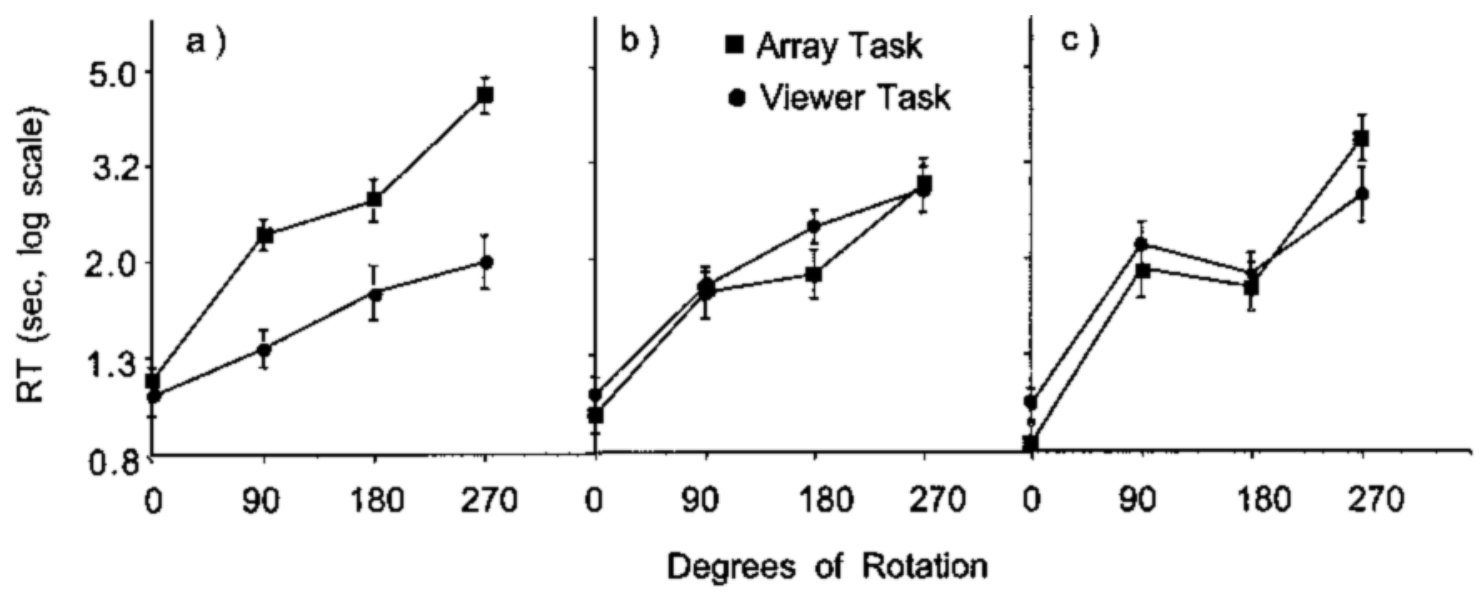

Figure 2. Log transformation graphs of the array and viewer mean reaction times. 


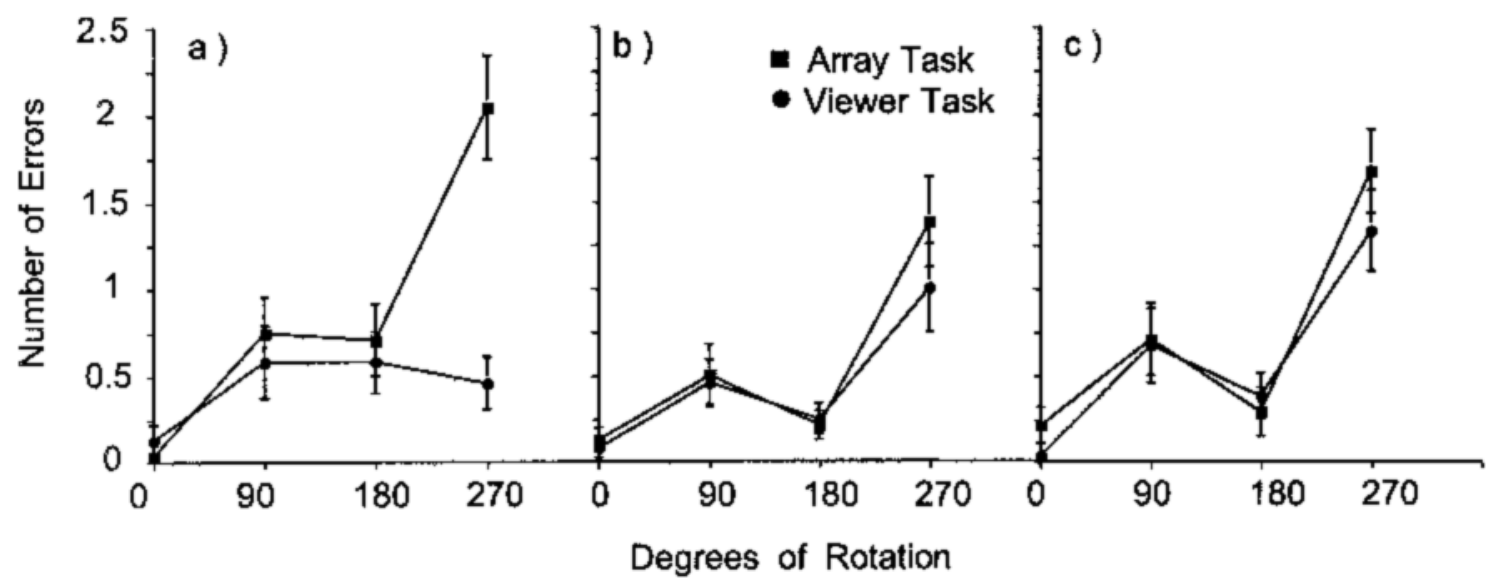

Figure 3. Graph of the mean number of viewer and array errors.

positions of the balls relative to the participant were top, bottom, right, and left. In the viewer task, participants imagined themselves rotating in the coronal plane in a motion similar to a cartwheel to their right (clockwise) or to their left (counterclockwise) in the center of the array. In the array task, participants were instructed to imagine the array rotating around them in a circle like a pinwheel, either to the left or to the right.

\section{Results and Discussion}

The major findings in Experiment 2 contrasted with the Experiment 1 results. Array and viewer task performance was not different in either number of errors or RTs. Position comparisons revealed an advantage for identifying objects in top/bottom positions faster than objects in right/left positions.

Latency. In contrast to the results of Experiment 1, in Experiment 2 there was no difference between participants' RTs in the array and viewer tasks. See Figure 2, panel b. One extreme outlier was replaced with the appropriate mean in the array task. There was no difference in overall mean latency between the array task $(M=$ $2.16 \mathrm{sec})$ and the viewer task $(M=2.19 \mathrm{sec})$. The ANOVA revealed a main effect of degree $[F(3,48)=63.03, p<$ $.0001]$, but no main effect of task. Analysis yielded a significant task $\times$ task order interaction $[F(1,16)=7.48$, $p=.015]$. A viewer $\times$ task order analysis indicated that participants responded more quickly during viewer trials when the viewer task was performed first than when the array task was performed first $[F(1,22)=7.39, p=.013]$. These task order findings were not replicated in any other latency results.

Errors. Levels of accuracy were the same in the coronal viewer and array tasks. In both tasks, the number of errors increased from $0^{\circ}$ to $90^{\circ}$, decreased from $90^{\circ}$ to $180^{\circ}$, then increased again at $270^{\circ}$. The overall mean number of errors was no different for the viewer $(M=$ 0.45 errors) and the array tasks $(M=0.56$ errors $)$. See Figure 3, panel b. The ANOVA revealed a main effect of degree $[F(3,48)=12.18, p<.0001]$. There was a significant task order $\times$ task interaction $[F(1,16)=12.33$, $p=.003]$ and a significant task order $\times$ degree interaction $[F(3,48)=3.78, p=.016]$.

Presson (1982) and Wraga et al. (2000) reported a pattern of error results in their transverse-plane array experiments that was similar to the current coronal-plane error findings. Fewer errors were committed at $180^{\circ}$ than at $90^{\circ}$ or $270^{\circ}$. They concluded that participants were simply reversing the positions of objects in the array rather than imagining a holistic array rotation. In the present experiment, participants may have done the same.

Position comparisons. RTs and number of errors were collapsed over positions relative to the location of the participant after a given imagined rotation (right/left and top/bottom) for the array and viewer tasks.

Latency. Top/bottom responses were faster than right/ left responses in both the viewer [top/bottom, $M=2.09$ sec; right/left, $M=2.23 \mathrm{sec} ; F(1,23)=4.21, p=.050]$ and the array task [top/bottom, $M=1.89 \mathrm{sec}$; right/left, $M=2.25 \sec ; F(1,23)=7.82, p=.010]$.

Errors. Position analy sis comparing mean numbers of top/bottom and right/left errors revealed no main effects.

\section{EXPERIMENT 3}

In Experiment 3, participants performed viewer and array mental rotations on a group of objects located in their median sagittal or right/left plane. The motivation for the experiment was the same as in Experiment 2; to determine whether the viewer mental rotation advantage is specific to the horizontal plane, this time by comparing imagined rotations in the sagittal plane.

\section{Method}

Participants. Twenty-four undergraduates participated (13 females, 11 males). Three additional participants were excluded from the study because they responded incorrectly to more than $50 \%$ of the test questions, and 1 participant was excluded for not following instructions.

Materials. The same array orientation as that used in Experiment 2 was employed. 
Procedure. In the sagittal experiment, participants stood with their feet placed on either side of the ball on the floor, facing one ball, with one behind them, and one overhead so that the array was in participants' median sagittal plane. The positions of the balls relative to the participants were front, back, top, and bottom. In the viewer task, participants mentally rotated themselves in the sagittal plane by imagining a motion similar to a front or a back flip in the center of the array. In the array task, participants were instructed to imagine the array rotating around them in a circle like a Ferris wheel, either forward or backward.

\section{Results and Discussion}

The viewer task was no easier than the array task when performed in the sagittal plane. The results of Experiment 3 replicated the Experiment 2 findings and not those of Experiment 1. Results of the position comparison were inconclusive.

Latency. The sagittal viewer and array task results were similar to the findings in Experiment 2. There was no difference in RTs to questions presented in the viewer and array tasks. The function of the array and viewer RTs did not show a linear increase with increase in degrees of rotation. Instead, $90^{\circ}$ rotation responses were no different from $180^{\circ}$ rotation responses. (See Figure 2, panel c.) One extreme outlier was replaced with the group mean for the appropriate degree of rotation in both the viewer and the array tasks. The overall mean RTs were not significantly greater in the array task $(M=2.33 \mathrm{sec})$ than in the viewer task $(M=2.19 \mathrm{sec})$. The ANOVA yielded a main effect of degree $[F(3,48)=48.68, p<.0001]$, but no other main effects.

Errors. The major error results in Experiment 3 mirrored the Experiment 2 findings; there was no difference between viewer and array errors, and a greater number of errors were committed during $90^{\circ}$ and $270^{\circ}$ rotations than during $0^{\circ}$ and $180^{\circ}$ rotations in both tasks. (See Figure 3, panel c.) There was no difference in the overall accuracy between the array task $(M=0.72 \mathrm{sec})$ and the viewer task $(M=0.61 \mathrm{sec})$. The ANOVA yielded a main effect of degree $[F(3,48)=24.91, p<.0001]$ and no other main effects or significant interactions.

Position comparisons. RTs and number of errors were collapsed over positions relative to the location of the participant with respect to the array after a given imagined rotation (front/back and top/bottom) for the array and viewer tasks.

Latency. There was no main effect of position.

Errors. Analysis revealed a main effect of position in the viewer task $[F(1,23)=11.02, p=.003]$, but no effect in the array task (top/bottom, $M=1.25$ errors; front/ back, $M=1.63$ errors). In the viewer task, front/back responses $(M=0.92$ errors $)$ were more accurate than top/bottom responses ( $M=1.50$ errors).

\section{GENERAL DISCUSSION}

When people are in a transverse relationship to the relevant environment and they try to imagine an object or a scene from a viewpoint different from their own, they can more effectively imagine themselves moving to that position than imagine the environment moving around them. However, this is not true when people imagine themselves performing rotations in the coronal and median sagittal planes. In these instances, they find no advantage in imagining that they are rotating rather than imagining the world rotating around them.

In our first experiment, participants performed imagined transverse plane rotations in the horizontal plane more quickly and accurately than imagined rotations of objects in their environment: These findings replicate those of previous studies (Amorim \& Stucchi, 1997; Huttenlocher \& Presson, 1979; Presson, 1982; Wraga et al., 2000). The explanation drawn from the results of these experiments was that mental manipulations of the egocentric reference frame, used in performing imagined observer rotations, were more successful than imagined transformations of the object-relative frame, used in performing imagined environmental rotations (Presson, 1982; Wraga et al., 2000). However, results of our experiments indicated that when participants were asked to imagine themselves or objects rotating in planes in which they had no experience locomoting (either in the coronal or in the sagittal plane), observer rotations were no easier to imagine than array rotations. Therefore, the proposal that egocentric representation always provides an advantage for imagined rotations is not generalizable to imagined rotations in the midsagittal plane or in the picture plane.

Position comparisons revealed that in all three experiments, speed and (to a lesser extent) accuracy of responses were largely dependent on the position of the requested response object. More specifically, objects in front/back and top/bottom positions were easier to identify than items in the right/left positions (i.e., when participants were asked "What is on the top/bottom?" they responded faster and more accurately than when asked "What is on the right/left?"). In Experiment 1, participants responded more quickly and accurately to front/back questions than to right/left questions. The findings replicate Wraga et al.'s (2000) results, which also indicated a front/back advantage over right/left question responses. In addition, RTs were smaller for top/bottom questions than for right/left questions in Experiment 2 . There was no difference between the latency and number of errors for top/bottom and front/back responses in Experiment 3.

These findings lend support to a number of studies showing that people encounter difficulty in responding to right/left discrimination tasks (Franklin \& Tversky, 1990; Parsons, 1987; Rigal, 1996; Roberts \& Aman, 1993). Franklin and Tversky found that when standing participants were asked to name objects placed to their right or left, in front or in back, above or below them, they took longest to respond to right/left questions, followed by front/back questions, and they were fastest at responding to top/bottom questions. Franklin and Tversky concluded that the results supported the spatial framework model, which states that "space is conceptualized 
in terms of three axes whose accessibility depends on body asymmetries and the relation of the body to the world" (p. 63).

Our results tie in well with an early Parsons (1987) experiment investigating imagined viewer rotations in different planes (around 13 axes, including the 3 major axes). Participants imagined themselves rotating into the position of a human model pictured at various orientations. They said "now" when they had completed the mental rotation. Parsons found that transformations in the transverse plane were performed more rapidly than transformations around oblique axes or in the coronal or sagittal planes of the observer. This finding provides important evidence for interpreting the results of the present experiments because it directly compared viewer rotation tasks within subjects. It allows us to conclude that viewer transverse rotations are performed more efficiently than rotations in other planes rather than showing that array transverse rotations are more difficult than array rotations around other axes. Overall, the present experiments revealed that there is no advantage for imagined egocentric versus array rotations for objects located in an observer's coronal and sagittal planes.

There are three accounts that could explain the special nature of imagined rotations in the transverse plane. These accounts make appeals to physics, orthogonality, or experience. First, egocentric rotations may be easier only for imagined rotations that are physically possible. Because of gravity acting on our bodies, people can move easily in the transverse plane but not the other two. Alternatively, the egocentric advantage may be specific to the orthogonal (perpendicular) relationship of the array to the observer. All that may be required to observe a viewer advantage is an orthogonal relationship between the principal axis of the observer and the imagined rotation relative to the array. Since our participants were always standing, horizontality and orthogonality were confounded. Finally, if imagined rotations are procedures that people use to prepare for intended movements in the environment, then experience with performing imagined or actual rotations in the horizontal plane may cause people to become more efficient at imagining rotations in this plane. Because they have no such practice in the sagittal or coronal plane, there is no advantage for imagining egocentric rather than environmental rotations in these planes. These explanations are not mutually exclusive. Experience, gravity, and orthogonality together, or a combination of any two could produce the results seen in the present experiments.

Subsequent to the present experiments, Creem, Wraga, and Proffitt (in press) investigated the exact cause of the transverse-plane viewer advantage. They compared viewer and array imagined rotations performed by prone participants, and performed on arrays not perpendicular to the participants' principal axis. Participants were asked first to imagine themselves orthogonal to the array and then to perform viewer and array rotation tasks.
Creem et al. (in press) found a viewer advantage in all experiments in which participants imagined rotations orthogonal to their imagined orientation. Neither their actual location nor whether the rotation was physically possible diminished the viewer advantage. These findings indicate that the imagined plane of rotation of the array is more important in influencing viewer task advantage than is gravity. On the basis of the Creem et al. results, it seems likely that familiarity plays a major role in the viewer-relative transverse rotation advantage. Given our natural posture, mode of locomotion, and gravity, transverse rotations are ubiquitous. Self-rotations are rare. Creem et al. clearly showed that what matters is whether the axis of imagined rotation coincides with the body, not with gravity. On the other hand, it is possible that the body axis is special for some other reason unrelated to experience. While eliminating the gravity explanation, the Creem et al. studies cannot resolve whether the critical variable is orthogonality to the principle axis of the body or experience. We cannot imagine how to experimentally unconfound these two variables. ${ }^{2}$

Studies reveal that people are better at imagining themselves rotating in the world than they are at imagining the world rotating around them in the horizontal plane (Amorim \& Stucchi, 1997; Huttenlocher \& Presson, 1979; Presson, 1982; Wraga et al., 2000). However, these findings do not generalize to imagined rotations performed in all planes. The present experiments indicated that imagined observer rotations are equally as difficult as imagined rotations of the environment when people perform these rotations in the midsagittal plane or in the picture plane. The advantages of imagined viewer rotations are specific to mental rotations performed in the transverse plane.

\section{REFERENCES}

Alivisatos, B., \& Petrides, M. (1996). Functional activation of the human brain during mental rotation. Neuropsychologia, 35, 111-118. Amorim, M., \& Stucchi, N. (1997). Viewer- and object-centered mental explorations of an imagined environment are not equivalent. $\mathrm{Cog}$ nitive Brain Research, 5, 229-239.

Cohen, M. S., Kosslyn, S. M., Breiter, H. C., DiGirolamo, G. J., Thompson, W., Anderson, A. K., Bookheimer, S. Y., BelliVEAU, J., \& Rosen, B. (1996). Changes in cortical activity during mental rotation: A mapping study using functional magnetic resonance imaging. Brain, 119, 89-100.

Cooper, L. A. (1975). Mental rotation of random two-dimensional shapes. Cognitive Psychology, 7, 20-43.

Creem, S. H., Wraga, M., \& Proffitt, D. R. (in press). Imagining physically impossible self-rotations: Geometry is more important than gravity. Cognition.

Franklin, N., \& Tversky, B. (1990). Searching imagined environments. Journal of Experimental Psychology: General, 119, 63-76.

Huttenlocher, J., \& Presson, C. C. (1979). The coding and transformation of spatial information. Cognitive Psychology, 11, 375-394.

Kosslyn, S. M., DiGirolamo, G. J., Thompson, W. L., \& Alpert, N. M. (1998). Mental rotation of objects versus hands: Neural mechanisms revealed by positron emission tomography. Psychophysiology, 35, 151-161.

Milner, A. D., \& Goodale, M. A. (1995). The visual brain in action. New York: Oxford University Press. 
Parsons, L. M. (1987). Imagined spatial transformation of one's body. Journal of Experimental Psychology: General, 116, 172-191.

Parsons, L. M., Fox, P. T., Downs, J. H., Glass, T., Hirsch, T. B., Martin, C. C., Jerabek, P. A., \& Lancaster, J. L. (1995). Use of implicit motor imagery for visual shape discrimination as revealed by PET. Nature, 375, 54-58.

Presson, C. C. (1982). Strategies in spatial reasoning. Journal of Experimental Psychology: Learning, Memory, \& Cognition, 8, 243-251.

RIGAL, R (1996). Right-left orientation, mental rotation, and perspectivetaking: When can children imagine what people see from their own viewpoint? Perceptual \& Motor Skills, 83, 831-842.

Roberts, R. J., \& Aman, C. J. (1993). Developmental differences in giving directions: Spatial frames of reference and mental rotation. Child Development, 64, 1258-1270.

Shepard, R. N. (1994). Perceptual-cognitive universals as reflections of the world. Psychonomic Bulletin \& Review, 1, 2-28.

Shepard, R. N., \& Cooper, L. A. (1982). Mental images and their transformations. Cambridge, MA: MIT Press.

Shepard, R. N., \& Metzler, J. (1971). Mental rotation of threedimensional objects. Science, 171, 701-703.

Tukey, J. W. (1977). Exploratory data analysis. Reading, MA: AddisonWesley.

Wraga, M., Creem, S. H., \& Proffitt, D. R. (1999). The influence of spatial reference frames on imagined object- and viewer rotations. Acta Psychologica, 102, 247-264.
Wraga, M., Creem, S. H., \& Proffitt, D. R. (2000). Updating displays after imagined object and viewer rotations. Journal of Experimental Psychology: Learning, Memory, \& Cognition, 26, 151-168.

\section{NOTES}

1. Logarithmic and square root transformations are appropriate to perform in order to examine skewed RT and error data (Tukey, 1977), and the data were analyzed using these figures. However, to ensure the significance of our results, statistical analyses were performed on raw data prior to the transformations; all of the same significant results were found in both cases.

2. One could investigate the performance of a selective portion of the population with sagittal or coronal plane movement experience (such as gymnasts or astronauts) to attempt to separate the two variables. However, results would be informative only if they indicated a coronal or sagittal imagined viewer rotation advantage for experienced people. If results were no different than in our studies, it would be impossible to conclude that orthogonality alone is the deciding factor. Instead, it could be argued that the magnitude of transverse-plane experience is too great to provide a participant with enough experience to compete with a lifetime of transverse-plane practice.

(Manuscript received August 11, 1999; revision accepted for publication August 28, 2000.) 